\title{
DEVELOPMENT OF A DYNAMIC, RECONFIGURABLE FINGER-HAND SKELETAL STRUCTURE
}

\author{
Shreya Patki \\ Mechanical Automotive, and Materials Engineering \\ University of Windsor \\ Windsor, Canada \\ patki1@uwindsor.ca
}

\author{
R. Jill Urbanic \\ Mechanical Automotive, and Materials Engineering \\ University of Windsor \\ Windsor, Canada \\ jurbanic@uwindsor.ca
}

\begin{abstract}
A finger or hand brace may be required to be worn for people who require physical support or 'controlled motion' support. Ehlers Danlos Syndrome (EDS) patients have connective tissue disorders, and wear braces to support and limit motion. Manual assembly operators, carpal tunnel sufferers, and the elderly may also benefit from wearing finger or hand braces. Additive manufacturing (AM) solutions provide flexible manufacturing options for customized braces; however, a CAD model must be developed to facilitate a manufacturing solution. The goal of this research is to develop a readily adaptable CAD model for a hand, which would link to a flexible brace model. Rhinoceros ${ }^{\circledR}$ and Grasshopper ${ }^{\circledR}$ modelling tools are employed to develop a solution that can be dynamically manipulated to adjust to a specific hand size, and/or localized finger-hand configurations. The final CAD model (hand or brace) can then be built using an AM process.
\end{abstract}

Keywords-human skeletal modeling; dynamic CAD model; finger; hand; additive manufacturing

\section{INTRODUCTION}

The human hand is an incredible tool. It is capable of up to $47 \mathrm{~kg}$ grip strength ${ }^{1}$ (with a $9.5-9.7 \mathrm{~kg}$ standard deviation for men aged $20-49$ ) [1] as well as controlling extremely fine motor movements. However, 'tools' can wear. Complex motions, such as securing a button, require dexterity and precise motor control. Strength and fine motor control may prove to be difficult for people with Ehlers Dowler Syndrome (EDS), a connective tissue disorder, for people who encounter repetitive strain injury when performing tasks that are prone to developing carpal tunnel syndrome (CTS), and the elderly.

For the people who suffer from EDS, everyday activities are problematic [2]. The braces for the fingers and/or hand are required to provide support, the needed limits for motion, and to allow for some precise motion control [3]. CTS is condition that causes pressure on the median nerve in the hand, which results in numbness in the hand, tingling, or itching. In severe cases, there is pain. CTS can occur as a result of repetitive motion activities where the wrist is overextended repeatedly. Other conditions linked with CTS are diabetes, high blood pressure, or fracture or trauma to the wrist. CTS is the most common nerve compression syndrome, affecting 3 to $6 \%$ of the American public [4]. As shown in Fig. 1, the manufacturing sector typically has $2-3$ times more injury occurrences compared to most other industries [5]. Well-designed custom braces would allow workers to perform their tasks with the needed dexterity and the required control while keeping the wrists in a neutral position and reducing the pressure on the nerves. People in the older demographic may also have lower dexterity [6] and have trouble with daily tasks such as holding cutlery and opening containers. Proper brace designs can assist with support and motion control, similar to EDS patients, although the physical conditions are different.

Although hand braces may be useful for many situations, 'general' sized hand braces may not allow for the needed flexibility or comfort, and customized braces are costly. Localized customization may be required if a person has had broken bones or arthritis. The introduction of additive manufacturing (AM), or 3D printing, processes can lead to a better 'quality of life' solution for the various scenarios described here, as complex patient-specific designs can be readily fabricated. However, prior to fabricating a solution, a manifold or 'watertight' CAD model must be created. The brace model should be linked to a personalized hand model, which is challenging to create. Reverse engineering techniques may be employed to collect point cloud data, but many scans would have to be performed to collect all the shape data, and then point cloud registration, filtering, mesh development refinement, and the final surface modeling must be performed.

Blending surface patches is time consuming and may require several iterations for representing the base geometry [78]. Alternatively, a parametric or reconfigurable model can be created, and then adapted to suit based on selected measurements, which is the goal of this research. Creating an adaptable joint - finger - hand model, which allows for either global or local modifications without executing advanced surface or solid modelling commands manually, will facilitate the development of a buildable model, and any necessary design modifications determined from field testing or usage. Coupling the reconfigurable hand model to a brace model, and leveraging AM processes, will provide a platform for 
customizable, yet affordable, hand braces for the general public.

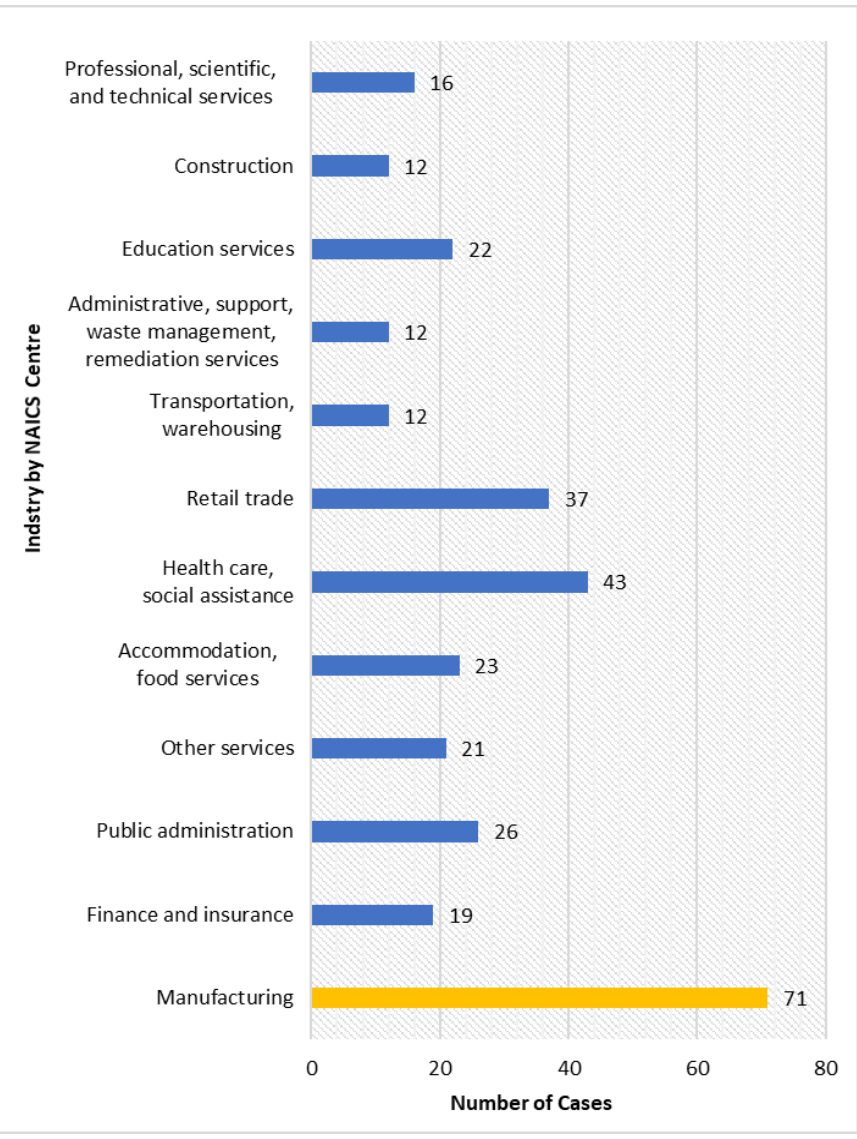

Figure 1. Carpal tunnel cases by North American Industry Classification System (NAICS) [5]

\section{BACKGROUND/LITERATURE}

Variations in the human hand/fingers size and shape are due to gender, age, body mass index (BMI), activity levels, and differences in body symmetry (i.e. the left and right hand and fingers will have differences). As illustrated by the anthropological data for the thumb and index finger (Table 1), there are significant variations from person to person (up to $35 \%$ for the thumb breadth), and these variations need to be considered when developing a customizable brace solution.

TABLE I. AVERAGE VALUES FOR SELECTED PERCENTILE DATA FOR THE THUMB AND INDEX FINGER, ADAPTED FROM [9]

\begin{tabular}{|l|l|c|c|c|c|c|}
\hline \multirow{2}{*}{ Dimension } & Gender & $\begin{array}{c}\text { 5th } \\
\text { percentile } \\
\text { (mm) }\end{array}$ & $\begin{array}{c}\text { 50th } \\
\text { percentile } \\
\text { (mm) }\end{array}$ & $\begin{array}{c}\text { 95th } \\
\text { percentile } \\
\text { (mm) }\end{array}$ & $\begin{array}{c}\Delta \text { 5th - } \\
\text { 95th } \\
\text { percentile }\end{array}$ & $\begin{array}{c}\text { Var. } \\
\text { from } \\
\text { mean }\end{array}$ \\
\hline \multirow{2}{*}{$\begin{array}{l}\text { Thumb } \\
\text { length }\end{array}$} & Male & 44 & 51 & 58 & $\mathbf{1 4}$ & $\mathbf{1 4 \%}$ \\
\cline { 2 - 8 } $\begin{array}{l}\text { Thumb } \\
\text { breadth }\end{array}$ & Female & 40 & 47 & 53 & $\mathbf{1 3}$ & $\mathbf{1 4 \%}$ \\
\cline { 2 - 8 } $\begin{array}{l}\text { Index finger } \\
\text { length }\end{array}$ & Female & $11-12$ & 23 & $26-27$ & $\mathbf{1 6}$ & $\mathbf{3 5 \%}$ \\
\cline { 2 - 8 } & Male & 64 & 72 & 79 & $\mathbf{1 4}$ & $\mathbf{3 4 \%}$ \\
\hline
\end{tabular}

Presently, the standardized models that have been created to date are constructed for one hand [10], and for the majority of the population - irregular or damaged hand types are not taken into consideration. However, using advanced reverse engineering techniques, specialized models can be very accurate when modeled to fit a custom hand type [11].

Ideally, a complex and detailed model, which can be easily modified, should be constructed. There are general 'structural' relationships that can be exploited linking finger geometry to hand length and width relationships (Fig. 2, Table II and III), and establishing a robust general model that allows for local modifications that could allow for rapid changes, would minimize the reverse engineering data collection.

The goal of this research is to leverage advanced CAD modeling techniques to create a dynamically reconfigurable hand model that can be animated to test finger movement, and how a brace would behave for different positions (ex. A fist or gripping position).

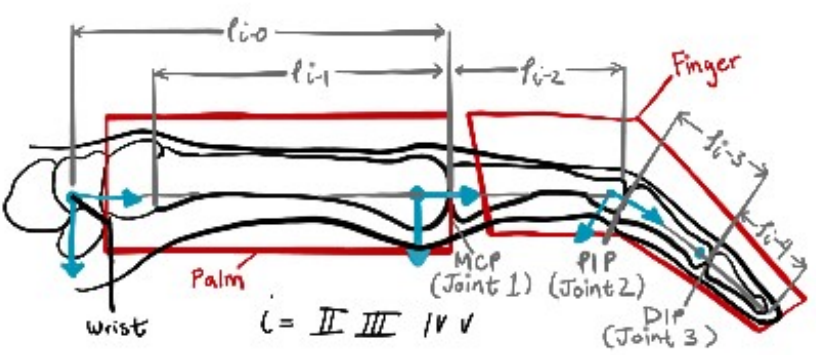

Figure 2. The 'i' represents each finger, the thumb being I and pinky V.

TABLE II. TABLE OF METACARPAL RELATIONSHIPS, WHERE $\mathrm{HL}=\mathrm{HAND}$ LENGTH [12]

\begin{tabular}{|c|l|}
\hline \multicolumn{2}{|l|}{ Metacarpal Bones } \\
\hline Thumb & $0.251 * \mathrm{HL}$ \\
\hline Index & $\left((0.374 * \mathrm{HL})^{2}+(0.126 * \mathrm{HB})^{2}\right)^{0.5}$ \\
\hline Middle & $0.373 * \mathrm{HL}$ \\
\hline Ring & $\left((0.336 * \mathrm{HL})^{2}+(0.077 * \mathrm{HB})^{2}\right)^{0.5}$ \\
\hline Pinky & $\left((0.295 * \mathrm{HL})^{2}+(0.179 * \mathrm{HB})^{2}\right)^{0.5}$ \\
\hline
\end{tabular}

TABLE III. TABLE OF FINGER PARAMETRIC RELATIONSHIPS FOR THE PHALANGEAL BONES[12]

\begin{tabular}{|c|c|c|c|}
\hline & Proximal & Middle & Distal \\
\hline Thumb & $0.196 * \mathrm{HL}$ & & $0.158 * \mathrm{HL}$ \\
\hline Index & $0.265 * \mathrm{HL}$ & $0.143 * \mathrm{HL}$ & $0.097 * \mathrm{HL}$ \\
\hline Middle & $0.277 * \mathrm{HL}$ & $0.170 * \mathrm{HL}$ & $0.108 * \mathrm{HL}$ \\
\hline Ring & $0.259 * \mathrm{HL}$ & $0.165 * \mathrm{HL}$ & $0.107 * \mathrm{HL}$ \\
\hline Pinky & $0.206 * \mathrm{HL}$ & $0.117 * \mathrm{HL}$ & $0.093 * \mathrm{HL}$ \\
\hline
\end{tabular}

\section{RESEARCH METHODOLOGY}

Initial research has been performed to design, fabricate, and test specialized designs for finger and hand braces [13]. Different parametric modelling approaches are being explored for readily accommodating special conditions. The modelling approach should be flexible, adaptable, easy to use by nondesigners, and require a minimal amount of rendering time. For this research, the 64-bit version of Rhinoceros ${ }^{\circledR}$ is employed with the Grasshopper ${ }^{\circledR}$ graphical programming and Bongo ${ }^{\circledR}$ animation add-ons. The Grasshopper ${ }^{\circledR}$ graphical programming tool can leverage known structural relationships and can

${ }^{1}$ Hand strength is measured by the quantity of static force a hand can squeeze around a dynamometer, and is measured in kg or lb. 
propagate changes throughout the whole design simultaneously. Local modifications can be performed using the Rhinoceros ${ }^{\circledR}$ CAD tools, and joint / motion relationships for any design can be readily modeled for virtual testing. The general process flow is shown in Fig. 3. The methodology presented is an overview. The details are not included due to space constraints.

The basic finger bone modelling is established for the thumb as it has the simplest structure (Table III). A simple model of the proximal thumb bone with flat ends and a tapered intermediate section was developed using multiple lofted circles. Since every hand is unique, the lengths of the fingers need to be calculated using the parametric relationships shown in Table I and II (i.e., using the hand length as the key input). This static model was updated to include the distal bone.

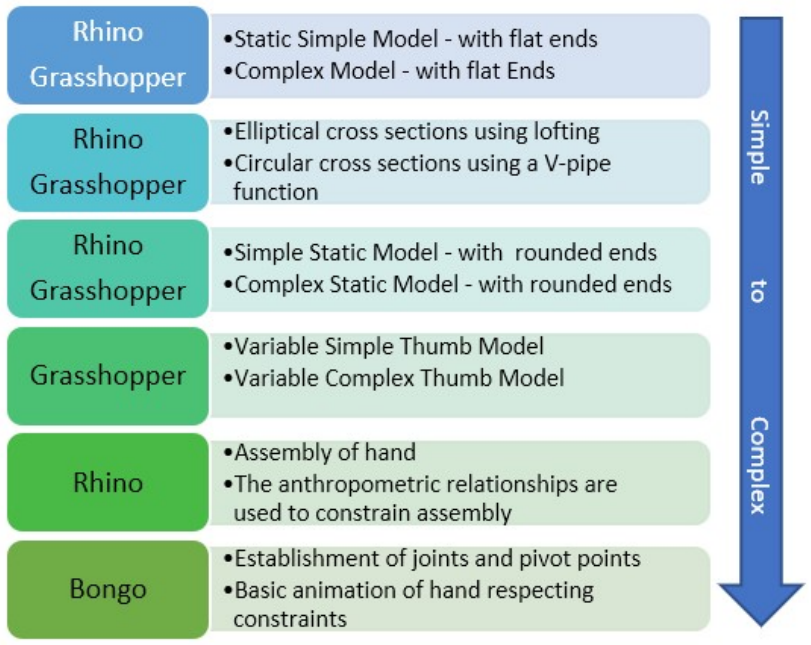

Figure 3. Steps in creating the final adaptable model

Once basic geometric relationships were established, elliptical cross-sections, with specialty Grasshopper ${ }^{\circledR}$ programming commands (V-Pipe or variable pipe), are employed to make this model more realistic. Sets of ellipses, and their control planes, are established. The model with the elliptical cross sections is more customizable as there are variable radii in two directions, and the ellipse positions may be altered using the input to the parametric relationships in the model (Fig. 4). Radii are controlled by a 'slider bar' input, as shown in Fig.8. This modeling approach leverages known relationships yet can accommodate of a wider variety of hands or localized variations, i.e., an arthritic hand, as shown Fig. 5.

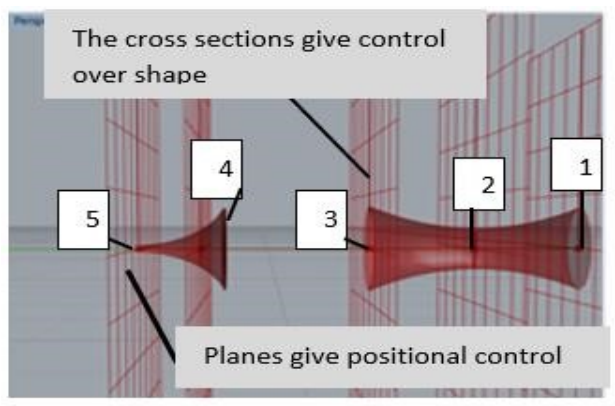

Figure 4. Elliptical cross sections and control planes illustrating the local control of the finger shapes. The 5 cross sections are labelled.

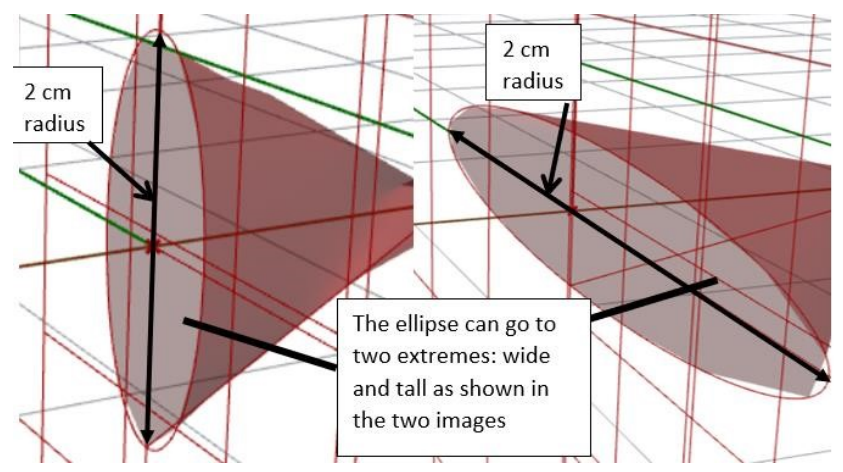

Figure 5. Another angle of the bone cross section and its flexability

Using the elliptical model as a base line, the rounded ends to the proximal bone are added via ellipsoids, where the control geometry is linked to the bone geometry. The endcaps model can also be personalized, as necessary. The progression from a bone to a finger is shown from right-top counter clockwise to right bottom (labelled 1-4) in Fig. 6. The semi-circular ring at the end of the finger is optional for now.

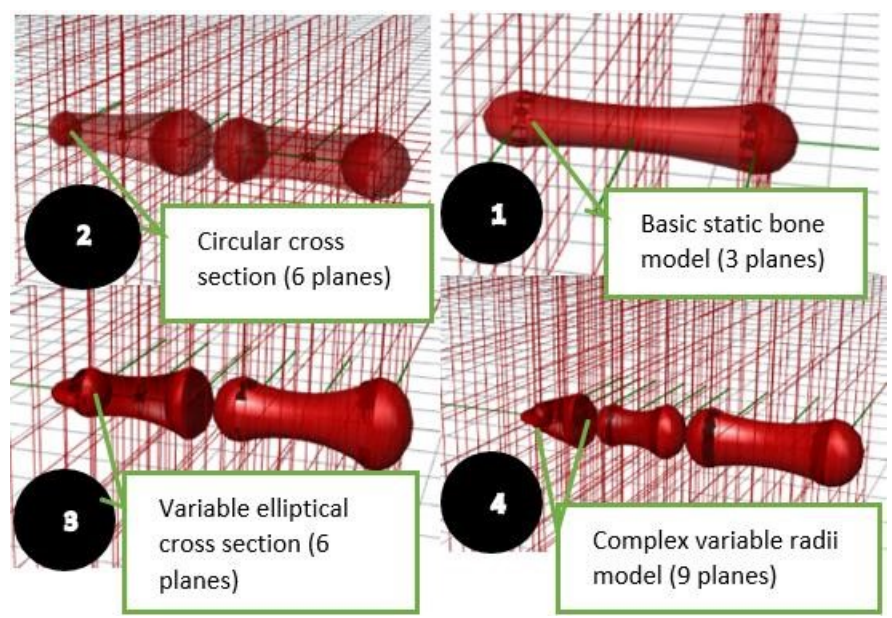

Figure 6. The build of the finger bones ordered from simple to complex (and order of creation)

Using the relationships and design approach, the bonefinger model is updated to become automatically adaptable. Instead of using the fixed numbers with the formulas and parametric relationships, 'slider bar' inputs or variables, which are controlled by the user, are utilized. The flexibility provided by the slider bars is shown in Fig. 7, 8 and 9.

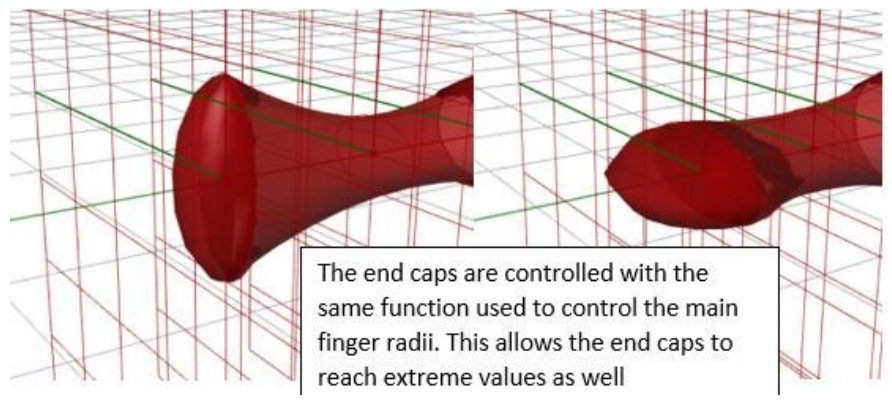

Figure 7. The adaptability of the end caps demonstrated 


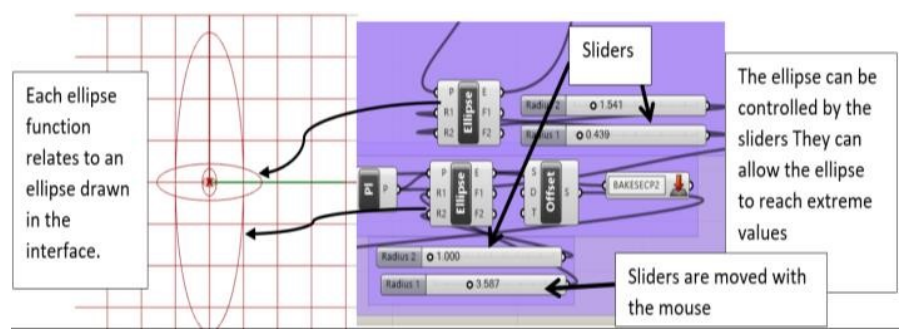

Figure 8. The simplicty of changing the finger radii is shown with the radii if the finger bone

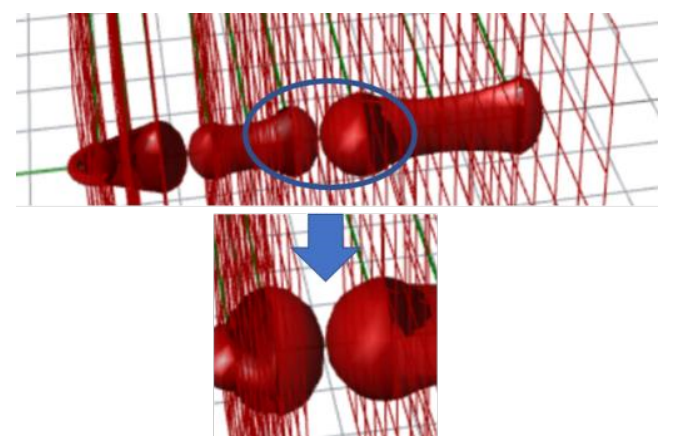

Figure 9. The wide range of the radii is demonstrated through the extreme sizes of the end caps shown.

The interface of the Grasshopper ${ }^{\circledR}$ algorithm is shown in Fig. 10. The parts are connected to each other using 'wires' in the program. This feature allows the input data to be transformed using mathematical functions, and the programmable control shapes to create surface, mesh, or solid model geometry in the Rhinoceros ${ }^{\circledR}$ environment.

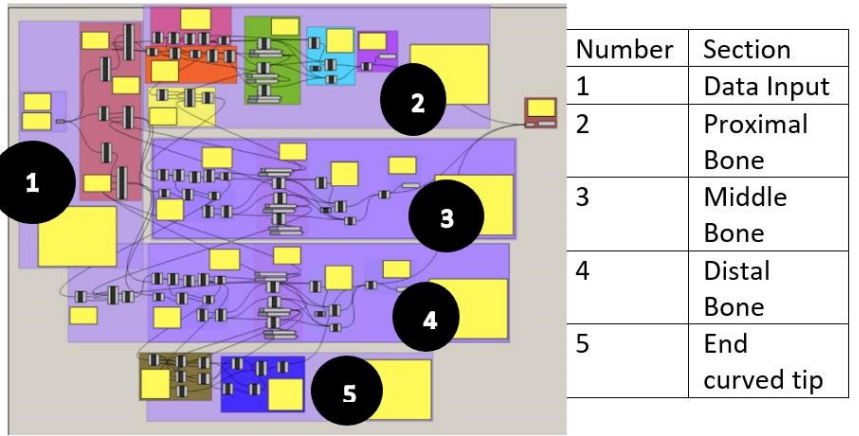

Figure 10. Labelled Grasshopper ${ }^{\circledR}$ interface shown (the behind the scenes of the finger model).

These finger models can easily be assembled into the individuals hand model (in the Rhino ${ }^{\circledR}$ environment) using anthropometric relationships and constraints to link the individual components into a final kinematic assembly. This bone-finger-hand model can then be animated for different situations such as hand gripping using the Bongo ${ }^{\circledR}$ add-on. Once the appropriate relationships are finalized, custom finger braces or hand brace models can be built on top of this.

\section{RESUlTS AND DISCUSSION}

The result of this process is a customizable bone-fingerhand model that can be adaptable for a variety of shapes instantaneously. The programming elements of this model allows a hand configuration to be programmed using parametric relationships established by other researchers. The hand length and breadth are measured. They are then used as an input to establish the bone lengths for the metacarpal and phalangeal bones each finger. Example solutions are shown in Figure 11 and 12. They are hand of a young female and young male. On the left is the wireframes printout of the model, center the hand, and on the right the 3D shaded model of the hand from Rhinoceros ${ }^{\circledR}$. The reconfigurable reverse engineered hand models are created.

Both hands shown have different hand lengths, breadths and finger radii, and this model was able to conform to the measurements and create a customized model of the hand.

A preliminary 'proof of concept' animation of these reconfigurable models has been completed. Examples of two animations with developed with the Bongo ${ }^{\circledR}$ add-on are shown in the links below (and Fig. 13 and 14).

https://www.screencast.com/t/m0Fec2dG2mE

https://www.screencast.com/t/qOnf6SetqA

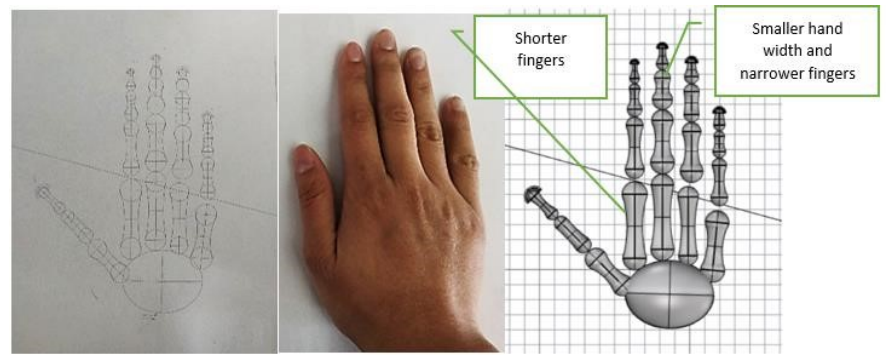

Figure 11. Female hand model.

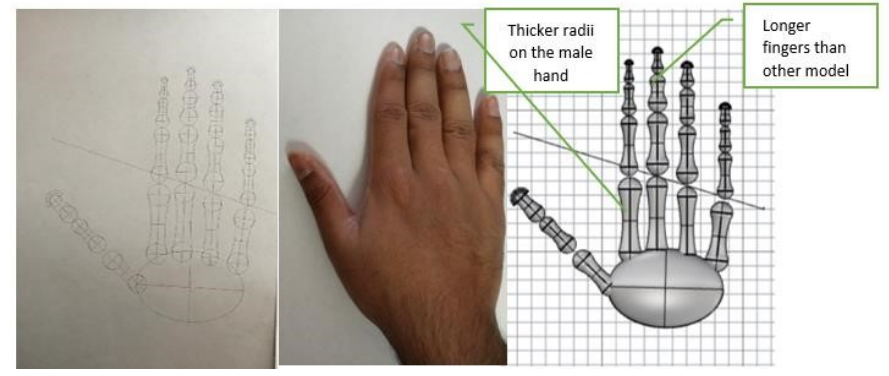

Figure 12. Male hand model

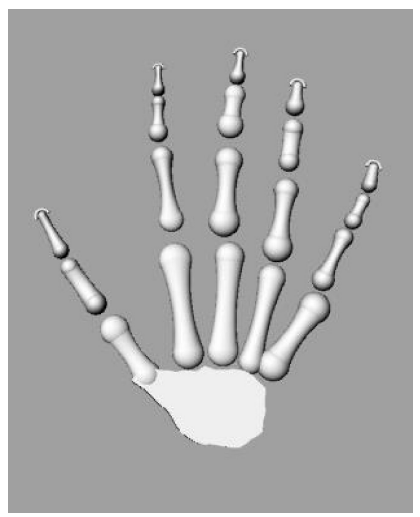

Figure 13. The hand model with moderately spread apart fingers 


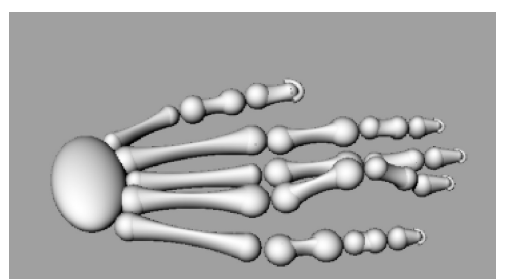

Figure 14. Still from a hand model animation of finger movement

\section{SUMMARY AND CONCLUSIONS}

Reverse engineering a hand to create an editable surface or solid model is a time intensive task and requires highly skilled designers familiar with surface modeling strategies. The goal to develop a dynamically reconfigurable bone-finger-hand model was realized using a set of modeling tools typically used in animation environments. Developing organic parametric models in CATIA is problematic due to linking the many bonefinger-hand constraints [14].

As human anatomy has general known relationships (anthropometric data), but individual variants, the Grasshopper ${ }^{\circledR}$ visual programming language is used to leverage the relationships to create editable models in the Rhinoceros environment. This foundational research allows for the creation a customized hand brace without a significant amount of measurements or data manipulation for developing a personalizing CAD model. This CAD model can then be used as an input for an AM process.

Throughout this process, the main goal was to add enough detail to represent the uniqueness of the individual's hand but not to be overly complex or have the data entry be a timeconsuming task. Due to the model's ability to have full control over the shape of the finger, (essentially the thickness), muscles and skin will be added in the future. The overall process for creating specialty braces shown in Fig. 15.

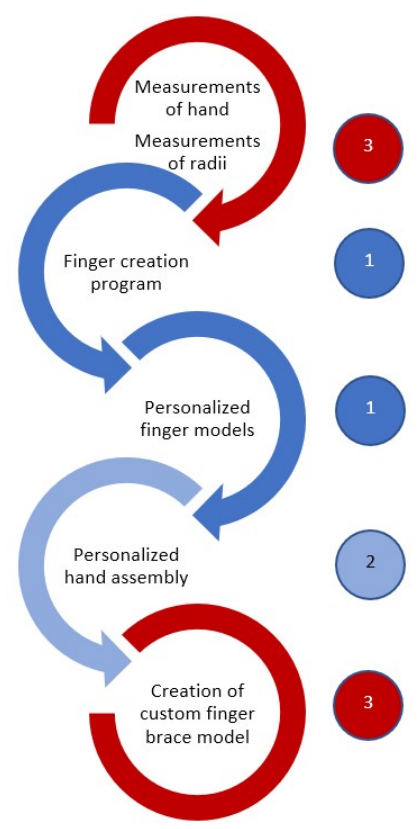

Figure 15. Steps for creating final adaptable hand and braces models (1 present research, 2 - on-going research, 3 - future work)

\section{VI. .FUTURE STUDIES}

Localized 'non-elliptical' geometry solutions will be explored. The animation models will be improved to include anthropometric relationships, as there are unique motion conditions for our hands. For example, all joints in the ring finger cannot be fully rotated inwards without creating motion in the middle or pinky fingers. The Grasshopper ${ }^{\circledR}$ model will be streamlined to add a user interface, data collection strategies determined, and brace design solutions will be designed, fabricated (using an AM process) and tested.

\section{ACKNOWLEDGMENTS}

This research is partially funded by the NSERC Discovery Grant and the University of Windsor Outstanding Scholars programs. Special thanks to hand model Swapnil Patki.

\section{REFERENCES}

[1] N. M Massy-Westropp, T. K. Gill, A. W. Taylor, R. W Bohannon, and C. L. Hill, "Hand grip strength: age and gender stratified normative data in a population-based study," BioMed Central, vol. 4, pp. 127-127, Apr. 2011.

[2] "Ehlers-Danlos syndrome," Mayo Clinic, 13-Oct-2017. [Online]. Available: https://www.mayoclinic.org/diseases-conditions/ehlersdanlos-syndrome/doctors-departments/ddc-20362171.

[3] J. R. Gravlee and D. J. Van Durme, "Braces and splints for musculoskeletal conditions.," American Family Physician, vol. 75, no. 1, pp. 342-348, Feb. 2011.

[4] K. E. LeBlanc and W. Cestia, "Carpal Tunnel Syndrome," American Family Physician, vol. 83, no. 8, pp. 952-958, Apr. 2011.

[5] S. E. Luckhaupt et.al , "Prevalence and work-relatedness of Carpal Tunnel Syndrome in the working population, United States, 2010 national health interview survey," American Journal of Industrial Medicine, vol. 56, no. 6, pp. 615-624, Apr. 2012.

[6] E. Carmeli, H. Patish, and R. Coleman, "The aging hand," The Journals of Gerontology: Series A, vol. 58, no. 2, pp. 146-152, Feb. 2003.

[7] M. Attene, M. Spagnuolo, "Automatic Surface Reconstruction from Point Sets in Space", Computer Graphics Forum, vol. 19, pp. $457-465$, 2000.

[8] L.M Galantucci, G. Percoco., Spina, R., 2004, "An artificial intelligence approach to registration of free-form shapes", CIRP Annals, vol. 53, no. 1 , pp. $139-142$.

[9] "Hand anthropometry," Ease of Use Assistant. [Online]. Available: http://usability.gtri.gatech.edu/eou_info/hand_anthro.php.

[10] M.I. Saludares and R. Atienza "Parametric 3D hand model",presented at the 7th International Conference on Intelligent Systems, Modelling and Simulation, Bangkok, Thailand 2016

[11] T. Rhee, U. Neumann, and J.P. Lewis "Human hand modelling from surface anatomy" Proceedings for 2006 ACM SIGGRAPH Symposium on Interactive 3D Graphics and Games.

[12] E. P. C. C. Pitarch, "Virtual human hand: grasping strategy and simulation," dissertation, Universitat Polit'ecnica de Catalunya (UPC), 2007.

[13] H. Kalemi, A. Khayat, R.J. Urbanic "Investigating exo-skeleton design and fabrication challenges for the hand region using material extrusion processes", in International Federation of Automatic Control Intelligent Manufacturing Systems, 2016, vol 49, pp 30-35.

[14] R.J. Urbanic, L. DiCecco, "Development of adaptable light weighting methods for material extrusion processes", American Society of Mechanical Engineers International Mechanical Engineering Congress \& Exposition, 2015 vol. 2A, pp. $1-3$ 